\title{
BOSWELLIA SERRATA, HARPAGOPHYTUM PROCUMBENS E CURCUMA LONGA NO MANEJO DA OSTEOARTRITE
}

\author{
Gisele Tedesco PALAVRO1; Rogério da Silva VEIGA². \\ ${ }^{1}$ Nutricionista, pós-graduada em Nutrição Clínica Funcional (VP- Unicsul) e pós-graduanda em Fitoterapia Inte-

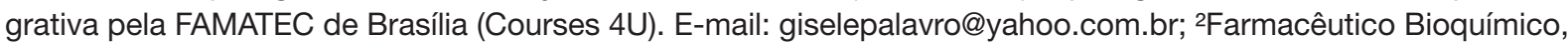 \\ Doutor em Ciências da Saúde na área de microbiologia do Departamento de Patologia da Faculdade de Ciências \\ Médicas da Santa Casa de São Paulo. E-mail: dasilvaveiga@uol.com.br
}

\section{Resumo}

A osteoartrite é um dos distúrbios do sistema osteoarticular mais comuns, chegando a atingir cerca de $15 \%$ da população mundial. Seu tratamento convencional é feito com medicamentos analgésicos e anti-inflamatórios que possuem risco de efeitos adversos, como ulcerações gastrointestinais, eventos hemorrágicos e nefrotoxicidade. Vislumbrando novas alternativas de tratamento, esta revisão buscou evidências científicas em estudos clínicos feitos em humanos com as espécies vegetais Boswellia serrata Roxb., Harpagophytum procumben DC. e Curcuma longa L. em pacientes com osteoartrite. Os resultados observados com o uso da $\mathrm{B}$. serrata foram alívio rápido da dor, redução da rigidez e recuperação da habilidade funcional articular, bem como sua segurança de uso em humanos, até mesmo por períodos prolongados. $\mathrm{O}$ extrato da cúrcuma-doméstica foi tão eficiente quanto o ibuprofeno no manejo da $\mathrm{AO}$ de joelho, os efeitos colaterais foram similares, porém com menos relatos de distúrbios gastrointestinais no grupo da cúrcuma-doméstica. O uso do Harpagophytum procumbens reduziu ou cessou o uso de analgésicos em $60 \%$ dos pacientes estudados, sendo comparado à diacereína. Os resultados encontrados nos apresentam um cenário muito promissor em se tratando de opções eficientes, acessíveis e seguras para o tratamento dos pacientes com problemas osteoarticulares, trazendo maior segurança de uso por serem plantas de uso tradicional e milenar em algumas culturas.

Palavras chave: Osteoartrite e plantas, osteoartrite e fitoterapia, harpagophytum procumbens e osteoartrite, boswellia e osteoartrite, curcuma e osteoartrite.

\section{Introdução}

Osteoartrite (OA) está entre uma das desordens crônicas mais comuns do sistema osteoarticular, chegando a afetar cerca de $15 \%$ da população mundial (BERENBAUM, F. 2008), resultando em progressivas alterações histológicas e sintomas debilitantes.

Caracterizada pelo surgimento da inflamação e estresse oxidativo, a osteoartrite leva a destruição da cartilagem articular e erosão dos ossos.
Induzida por citocinas pró-inflamatórias como a interleucina 1 (IL1), interleucina 6 (IL6) e fator de necrose tumoral $\alpha$ (TNF- $\alpha$ ), há um aumento da colagenase ou síntese de matriz metaloproteinase (MMP) e a degradação do colágeno tipo II, reduzindo a síntese dos inibidores de colagenase, colágeno e proteoglicanos (DRAGOS et al., 2017). A Organização Mundial da Saúde estimou em 2004 que a cada 1000 pessoas, 400 com idade acima de 70 anos sofrem com AO. Dessas 1000 pessoas, 800 possuem limitações de mobilidade nos graus 


\section{Brazilian Journal of Natural Sciences | Versão On-line ISSN 2595 - 0584 \\ Edição no 1- vol. 2 - maio 2018 | www.bjns.com.br}

moderado a severo, reduzindo drasticamente sua qualidade de vida (BERENBAUM, 2008).

Entre os fatores que contribuem para o aumento do risco de AO estão idade, sexo, sobrepeso, índice de massa corporal (IMC) aumentado, genética, etnicidade, dieta, trauma, atividades físicas e ocupacionais que implicam em estresse biomecânico das articulações (DRAGOS et al., 2017).

Seu tratamento médico convencional é feito com medicações antiinflamatória não esteroidais (AINES) e opióides, entre outras drogas que acabam acarretando em uma série de efeitos colaterais severos como ulcerações gastrointestinais, eventos hemorrágicos, nefrotoxicidade, etc. (MC ALINDON et al., 2014). Quanto maior o tempo de exposição a essas drogas e maior a idade do paciente, maiores são os riscos de efeitos colaterais (GUMBREVICIUS, MILASIUS, SVEIKATA, 2006). Objetivando a busca por um manejo terapêutico efetivo e que implique em menor risco de efeitos adversos para o paciente acometido da $\mathrm{AO}$, este artigo busca levantar evidências em humanos que respaldem o uso dos fitoterápicos na prática clínica.

\section{Metodologia}

A pesquisa compreende o levantamento bibliográfico de artigos publicados dentro do período de 2000 a 2017 disponíveis na base do Pubmed e Google acadêmico, utilizando-se os seguintes termos em inglês para busca de palavras chaves: "osteoarthritis and plants", "osteoarthritis and phytotherapy", "harpagophytum procumbens and osteoarthritis", "boswellia and osteoarthritis", "curcuma and osteoarthritis".

\section{Discussão}

\section{Boswellia serrata Roxb.}

É uma planta nativa da Índia, com uso popular há séculos na medicina ayurvédica, a goma resinosa extraída da árvore da Boswellia tem sido utilizada para o tratamento de problemas inflamatórios, particularmente na artrite (DRACOS et al., 2017).
Um estudo feito com 56 pacientes, divididos em 2 grupos, com idade entre 40 e 70 anos, buscou verificar a eficácia da Boswellia serrata Roxb. em pacientes com Osteoartrite. Ao grupo A $(n=29)$ foram ofertadas cápsulas com $6 \mathrm{~g}$ de resina de Boswellia serrata Roxb., divididos em 3 doses ao dia, administradas com água após a refeição, enquanto que o grupo $B(n=23)$ ingeriu as mesmas doses, na mesma frequência, acrescido de aplicação tópica de b Boswellia nas áreas afetadas. Ambos foram acompanhados por 8 semanas. Os resultados mostraram no grupo A: $11,54 \%$ dos pacientes relataram completa remissão, 15,38\% melhora marcante, 57,69\% melhora moderada, $11,54 \%$ melhora leve. No grupo B nenhum dos pacientes relatou completa remissão, 8,69\% melhora marcante, $69,56 \%$ melhora moderada e $21,74 \%$ relataram leve melhora. Ao avaliar os dados, verificou-se que o grupo A obteve melhor resultado (Boswellia via oral) que o grupo B (Boswellia via oral + uso tópico), o que levou a avaliação de que os pacientes do grupo A possuíam menor cronicidade e idade, junto de sintomas mais brandos, o que provavelmente influenciou os resultados (GUPTA et al., 2011).

Sengupta et al (2010) fez um estudo comparativo verificando a tolerância e eficácia do 5-Loxin ${ }^{\circ}$ e do Aflapin ${ }^{\circledR}$ contra a $\mathrm{AO}$ de joelho. Aflapin ${ }^{\circledast}$ é um produto derivado da goma resinosa da Boswellia serrata Roxb. padronizado em $20 \%$ de 3 -O-acetyl-11-keto-beta-boswellic acid (AKBA) enquanto que 5 -Loxin ${ }^{\otimes}$ é padronizado em $30 \%$ de AKBA.

AKBA foi identificado como o mais ativo componente do extrato da Boswellia e tem demonstrado ser um inibidor da 5-lipoxigenase (5-LOX), a enzima chave na biosintese dos leucotrienos do ácido araquidônico na cascata inflamatória celular (SAFAYHI et al., 1992).

O estudo duplo cego, randomizado, placebo controlado acompanhou 60 pacientes que receberam diariamente $100 \mathrm{mg}$ de 5 -Loxin ${ }^{\circledR}(\mathrm{n}=20)$ ou $100 \mathrm{mg}$ de Aflapin ${ }^{\circ}(\mathrm{n}=20)$ ou placebo $(\mathrm{n}=20) \mathrm{du}$ rante 90 dias. As avaliações aconteceram nos dias 7, 30, 60 e 90. No sétimo dia, observou-se melhora substancial nos parâmetros de dor no grupo Aflapin ${ }^{\circledR}$. Ao final do estudo, ocorreu uma melhora sig- 


\section{Brazilian Journal of Natural Sciences | Versão On-line ISSN 2595 - 0584 \\ Edição no 1- vol. 2 - maio 2018 | www.bjns.com.br}

nificativa em relação ao alívio da dor, habilidade física e qualidade de vida nos grupos Aflaxin ${ }^{\oplus} \mathrm{e}$ 5-loxin ${ }^{\oplus}$ em comparação ao placebo, tendo o grupo Aflaxin ${ }^{\oplus}$ demonstrado melhores resultados nos parâmetros de dor. Os parâmetros bioquímicos de sangue, urina e hematológicos coletados a cada visita, não apresentaram nenhuma alteração estatisticamente relevante ao longo do estudo. Nenhum efeito adverso severo foi reportado, sendo relatado apenas 2 casos de acidez, um do grupo Aflapin ${ }^{\circledR} \mathrm{e}$ outro do placebo (SENGUPTA et al., 2010).

Os mesmos pesquisadores do estudo anterior estenderam sua pesquisa através de outro estudo duplo cego, randomizado, placebo controlado que avaliou a velocidade de eficácia do Aflapin ${ }^{\circledR}$ em indivíduos com $\mathrm{AO}$ de joelho. Sessenta pacientes com $\mathrm{AO}$ de joelho nos graus leve a moderado foram selecionados e acompanhados durante 30 dias, recebendo $100 \mathrm{mg}$ de Aflapin ${ }^{\circledR}$ ou placebo diariamente. As avaliações partiram do dia 0 , dia 5 , dia 15 e dia 30. Com apenas 5 dias de uso, foi observado uma melhora significativa de $14,8 \%$ e $16,3 \%$ nos índices de VAS (escala analógica visual) e LFI (Lequesne's Functional Index) no grupo Aflapin ${ }^{\circ}$, demonstrando resposta rápida na redução da dor e na capacidade funcional da articulação (VISHAL, MISHRA, RAYCHAUDHURI, 2011). Os resultados observados nos estudos em humanos com OA tratados com B. serrata são promitentes, especialmente no alívio rápido da dor, redução da rigidez e recuperação da habilidade funcional articular, bem como sua segurança de uso em humanos, até mesmo por períodos prolongados (SENGUPTA, 2010).

\section{Curcuma longa L. (cúrcuma-doméstica)}

A Curcuma longa L., planta nativa da Ásia, é tradicionalmente conhecida por sua atividade anti-inflamatória. A curcumina é o extrato da Curcuma longa L. que concentra os curcuminóides, que são um grupo de compostos químicos que caracterizam a atuação bioquímica anti-inflamatória (AGGARWAL et al., 2006). A curcumina tem uma atividade anti-inflamatória comprovada em diversos estudos (JOE, B. VIJAYKUMAR, M. LOKESH,
BR. 2004), nos quais sua atuação se apresenta através da inibição de diversas moléculas inflamatórias como a fosfolipase, lipoxigenase, COX-2, leucotrienos, tromboxanos, prostaglandinas, óxido nítrico, colagenase, elastase, hialuronidase, fator de necrose tumoral e interleucina 12. (CHAINANI, 2003).

Em um estudo prospectivo randomizado que comparou os efeitos da curcumina e do diclofenaco de sódio, foram selecionados 80 pacientes com osteoartrite de joelho, divididos em 2 grupos. $\mathrm{O}$ primeiro grupo $(\mathrm{n}=34)$ recebeu $30 \mathrm{mg}$ de curcuminóides, 3 vezes ao dia, enquanto que o segundo grupo (n=39) recebeu $25 \mathrm{mg}$ de diclofenaco de sódio, na mesma frequência de uso. $\mathrm{O}$ estudo durou 4 semanas, a média de idade entre os pacientes foi de 64 anos. Para avaliar os resultados, os pacientes passaram por uma aspiração de cartilagem de joelho no início do estudo e outra ao final, com o objetivo de verificar os níveis de COX-2 secretados pelos monócitos do líquido sinuvial. Ambos os grupos obtiveram uma redução significativa nos níveis da enzima COX-2 pelos monócitos do fluído sinovial, não representando nenhuma diferença entre as duas terapias, sendo que os curcuminóides, além de ter custo menor, levaram a menos efeitos colaterais (NYOMAN et al., 2012).

Kuptniratsaikul et al (2014), conduziram um estudo randomizado, duplo-cego que comparou a eficácia e segurança do extrato da cúrcuma-doméstica e do ibuprofeno na redução da dor e na melhora da função da articulação de joelho. Foram selecionados 524 pacientes com AO de joelho, aos quais apenas 367 puderam participar do estudo.

No grupo A, 185 pacientes receberam $1.500 \mathrm{mg}$ de extrato de cúrcuma-doméstica, enquanto que 182 pacientes do grupo B receberam $1.200 \mathrm{mg}$ de ibuprofeno, ambos na forma de duas cápsulas após as refeições, $3 \mathrm{x}$ ao dia, durante 4 semanas. A taxa de melhora global em ambos os grupos foi de $64 \%$, não demonstrando diferença significativa entre os pacientes tratados com cúrcuma-doméstica a e com ibuprofeno. Os pesquisadores concluíram que o extrato de cúrcuma-doméstica foi tão eficiente quanto o ibuprofeno no manejo da $\mathrm{AO}$ de joelho, os efeitos colaterais foram similares, porém 


\section{Brazilian Journal of Natural Sciences | Versão On-line ISSN 2595 - 0584 \\ Edição no 1- vol. 2 - maio 2018 | www.bjns.com.br}

com menos relatos de distúrbios gastrointestinais no grupo da cúrcuma-doméstica.

\section{Harpagophytum procumbens DC.}

Tradicionalmente conhecida como "garra do diabo", essa planta de origem africana está presente em diversas farmacopéias, que a indicam como analgésica, estimulante das enzimas gástricas e digestivas, diurética e sedativa, estimulante do apetite e auxiliar nas desordens do sistema músculo esquelético (ALTERNATIVE MEDICINE REVIEW, 2008).

Em estudo duplo-cego e randomizado que comparou a tolerância e eficácia do Harpadol ${ }^{\circ}$ (extrato padronizado de Harpagophytum procumbens DC.) e da diacereína, 122 pacientes com osteoartrite de joelho ou quadril foram divididos em dois grupos: Harpadol ${ }^{\oplus}, 2.610 \mathrm{mg} / \mathrm{dia}$ e Diacereína, $100 \mathrm{mg} /$ dia. O estudo teve a duração de 4 meses. As escalas de dor espontânea mostraram melhora significativa em ambos os grupos. Houve um avanço da capacidade funcional não mostrando nenhuma diferença estatística nos grupos Harpadol ${ }^{\circledR}$ e Diacereína. Foram relatados poucos casos de reações adversas, sendo a diarréia a mais frequente $\left(8,1 \%\right.$ no grupo Harpadol ${ }^{\circ}$ e $26,7 \%$ no grupo diacereína). O uso de medicações anti-inflamatórias e analgésicas foi menor no grupo Harpadol $^{\circ}$ (CHANTRE, 2000).

Um grupo de pesquisadores do Reino Unido fez um estudo aberto que avaliou 259 pacientes com desordens reumáticas classificadas como suave ou moderada, em uma ou mais partes do corpo, tendo apresentado dor em pelo menos 2 dias da semana, pelas últimas 8 semanas.

A dose de comprimidos da tintura de Harpagophytum procumbens DC. ofertada aos voluntários foi de $960 \mathrm{mg} / \mathrm{dia}$, tomadas $2 \mathrm{x}$ ao dia, antes das refeições. Os voluntários foram acompanhados por 2 meses, com 3 visitas programadas, uma no dia 0 , outra no meio (semana 4 ) e outra ao final (semana 8). A eficácia da terapêutica foi avaliada através de 2 protocólos, the Western Ontario and McMasters Universities Osteoarthritis (WOMAC) Index and the Algofunctional Hand Osteoarthritis
Index. A tolerância foi mensurada através de relatos pessoais, análise sanguínea e testes de função hepática. A qualidade de vida foi mensurada pelo questionário SF-12. Houve uma melhora significativa $(>0,0001)$ nas escalas de dor geral, rigidez e função. Os índices de qualidade de vida aumentaram ao longo do estudo e $60 \%$ dos pacientes reduziram ou pararam de usar concomitantemente medicações para a dor. Em relação aos efeitos adversos, $17 \%$ dos pacientes relataram algum evento com provável relação com o Harpagophytum, em sua maioria, desconfortos gastrointestinais (WARNOCK, 2007). Sintomas gastrointestinais são os efeitos comumente relatados com o uso do Harpagophytum, sendo contraindicado em pacientes com úlceras gastroduodenais, diabetes e cálculos biliares (SETTY; SIGAL, 2005), observando que a maioria dos estudos tem colocado como fator de exclusão quaisquer dessas complicações. Importante observar que não foram relatados efeitos graves, apenas suaves ou moderados. O grau de tolerância relatado pelo paciente em $87 \%$ dos casos foi bom (WARNOCK, 2007).

$\mathrm{O}$ alívio da dor no paciente é o fator pontual mais importante que o profissional da saúde busca (SINGER et al., 2000), sendo importante observarmos que o uso do Harpagophytum reduziu ou cessou o uso de analgésicos em $60 \%$ dos pacientes estudados por Warnock et al (2007), sendo comparado à diacereína (CHANTRE, 2000). A melhora funcional e a redução da rigidez articular também foram fatores estatisticamente relevantes nos estudos apresentados nesta revisão, pontuando um progresso importante na qualidade de vida dos indivíduos.

\section{Conclusão}

Ao verificarmos como a osteoartrite afeta a qualidade de vida das pessoas, sendo uma das principais causas de limitação e incapacidade motora, buscar alternativas eficazes e seguras para o tratamento dessa desordem é de suma importância. Devido às limitações que os anti-inflamatórios convencionais apresentam, especialmente em relação aos efeitos adversos, precisamos reconhecer 


\section{Brazilian Journal of Natural Sciences | Versão On-line ISSN 2595 - 0584 \\ Edição no 1- vol. 2 - maio 2018 | www.bjns.com.br}

que a fitoterapia tem sido uma alternativa promissora. $\mathrm{O}$ uso tradicional e milenar das plantas merece nossa atenção, podendo nos indicar um caminho importante em relação às pesquisas científicas, que devem buscar alternativas seguras, acessíveis e eficazes para auxiliar o paciente reumatológico. Essa revisão teve como objetivo agrupar estudos que ampliem nossa visão sobre a fitoterapia na osteoartrite, no entanto mais estudos são necessários para que a investigação siga em busca de melhores escolhas no tratamento da osteoartrite.

\section{Referências bibliográficas}

AGGARWAL, B.B.; BHATT, D.I.; ICHIKAWA, H.; AHN, S.K.; SETHI, G.; SANDUR, K.S.; NATARAJAN, C.; SEERAM, N.; SHISHODIA, S. Chemical composition of turmeric in curcumin-biological and medical properties, 2006.

BERGMARK, S.; MESA, M D.; GIL, A. Plantderived health - the effects of turmeric and curcuminoids. Nutr Hosp. v.3, n.24, p.273-281, 2009.

CHAINANI, N. Safety and anti-inflamatory activity of curcumin: a component of turmeric (curcuma longa). J Compl Med, p.161-168, 2003.

CHANTRE, P.; CAPPELAERE, A.; LEBLAN, D.; GUEDON, D.; VANDERMANDER, J.; FOURNIE, B. Efficacy and tolerance of Harpagophytum procumbens versus diacerhein in treatment of osteoarthritis. Phytomedicine, n.7, v.3, p.77-83, 2000.

DRAGOS, D.; GILCA, M.; GAMAN, L.; VLAD, A.; IOSIF, L.; STOIAN, I.; LUPESCU, O. Phytomedicine in joint disorders. Nutrients, v.9, n.70, dez.2016/jan.2017.

GHASEMIAN, M.; OWLIA, S.; OWLIA, M.B. Review of Anti-Inflammatory Herbal Medicines. Advances in Pharmacological Sciences, p.1-11, 2016.

GUMBREVICIUS, G.; MILASIUS, A.; SVEIKATA, A. Nonsteroidal anti-inflamatory agents: choice between disturbances of gastrointestinal tract and cardiovascular toxicityKaunas). 2006; 42(5): 429-439.

GUPTA, P.K.; SAMARAKOON S.M.S.; CHANDOLA H.M.; RAVISHANKAR B. Clinical Evaluation of Boswellia serrata (Shallaki) resin in the management of sandhivata (osteoarthritis). AYU, v.32, n.4, oct-dec 2011. Disponível em: <https://www.ncbi.nlm.nih.gov/pmc/articles/ PMC3361921>. Acesso em: 9 ago, 2017.

HARPAGOPHYTUM PROCUMBENS (DEVIL'S CLAW), Alternative Medicine Review, v.13, n.3, 2008. Disponível em http://www.altmedrev. com/publications/13/3/248.pdf >. Acesso em 13 ago. 2017.

JOE, B.; VIJAYKUMAR, M.; LOKESH, BR. Biological properties of curcumin, cellular and molecular mechanisms of action. Critic Rev Food Science Nut, n.44, p. 97-111, 2004.

KUPTNIRATSAIKUL, V.; DAJPRATHAM, P.; MONTANA, W.T. et al. Efficacy and safety of curcuma domestica extracts compared with ibuprofen in patients with knee osteoarthritis: a multicenter study. Clinical Interventions in aging, n.9, p.451-458, 2014.

MCALINDON, T.E.; BANNURU, R.R.; SULLIVAN, M.C.; ARDEN, N.K.; BERENBAUM, F.; BIERMA-ZEINSTRA, S.M.; HAWKER, G.A.; HENROTIN, Y.; HUNTER, D.J.; KAWAGUCHI, $\mathrm{H}$. et al. OARSI guidelines for the non-surgical management of knee osteoarthritis. Osteoarthr Cartil, n.22, p. 363-388, 2014.

NYOMAN, K.; AHMAD, H.; WASILAH, R.; MARSETYAWAN. Ability of curcuminoid compared to diclofenac sodium in reduction the secretion of cycloxygenase- 2 enzyme by synovial fluid's monocytes of pacients with osteoarthritis. The Indonesian Journal of Internal Medicine, v.44, n.2, abril 2012.

SAFAYHI, H.; MACK, T.; SABIERAJ, J.; ANAZODO, M.I.; SUBRAMANIAN, L.R.; AMMON, H.P.T. Boswellic acids: novel, specific, nonredox inhibitors of 5-lipoxygenase. J Pharmacol. Exp. Ther, n.26, p.1143-1146, 1992.

SETTY, A.R.; SIGAL, L.H. Herbal medications commonly used in the practice of rheumatology: mechanisms of action, efficacy and side effects. Seminars in Arthritis and rheumatism, v.34, n.6, p.773-784, 2005.

SIDDIQUI, M. Z. Boswellia Serrata, a potential antiinflammatory agent: an overview. Indian J Pharm Sci, v.3, n.73, p. 255-261, may-jun. 2011. 
SINGER, F; MAYRHOFER, F.; KLEIN, G.; HAWEL, R.; KOLLENS, C.J. Evaluation of the efficacy and dose-response relationship of dexibuprofen in patients with osteoarthritis of the hip and comparison with racemic ibuprofen using the WOMAC osteoarthritis index. Int J Clin Pharmacol Ther, n.38, p15-24, 2000.

VILANUEVA, J.R.; ESTEBAN J.M.; VILLANUEVA, L.R. Solving the puzzle: what is behind our forefathers' anti-inflammatory remedies? J Intercultethnopharmacol, v.6, i.1, p.128-143, 2017.

VISHAL, A.A.; MISHRA, A.; RAYCHAUDHURI, P.S. A double blind, randomized, placebo controlled clinical study evaluates the early efficacy of Aflapin in subjects with osteoarthritis of knee. International Journal of Medical Sciences, n.8, 2011.

WAENOCK, M.; MCBEAN, D.; SUTER, A.; TAN, J.; WHITTAKER, P. Effectiveness and safety of devil's claw tablets in patients with general rheumatic disorders. Phytoterapy research, res.21, p.1228-1233, 2007.

WORLD HEALTH ORGANIZATION. WHO Monographs on Selected Medicinal Plants, v.1, 1999. Disponível em: < http://apps.who.int/medicinedocs/en/d/Js2200e/14.html>. Acesso em: 16 ago, 2017. 